International Journal of Wireless \& Mobile Networks (IJWMN) Vol. 4, No. 2, April 2012

\title{
Extended Ad Hoc on Demand Distance Vector Local Repair Trial for MANET
}

\author{
P. Priya Naidu ${ }^{1}$ and Meenu Chawla ${ }^{2}$ \\ ${ }^{1}$ Associate System Engineer, IBM Pvt. Limited, India \\ Priya.cse020@gmail.com \\ ${ }^{2}$ Associate Professor, Department of computer Science MANIT, Bhopal, India \\ chawlamemanit.ac.in
}

\begin{abstract}
Adhoc On-demand Distance Vector (AODV) is a routing schema for delivering messages in a connected Mobile Adhoc Network (MANET). In MANETs, a set of nodes are used to route the data from source to destination and it is assumed that nodes are distributed over the entire region. Connectivity between any sources to destination pair in the network exists when they are in radio range of each other. The technique used to deal with the issues called Local Repair. Local Repair is an important issue in routing protocol which is needed for minimizing flooding and performance improvement. Local Repair is one of the major issues in the protocol; routes can be locally repaired by the node that detects the link break along the end to end path. Local Repair will increase the routing protocol performance. In this paper, the existing Local Repair Trial method in AODV is extended to achieve broadcasting and minimizing the flooding. The Enhanced protocol first creates the group of mobile nodes then broadcasting can be done and if the link breaks then local repair technique can be applied. In the network the numbers of intermediate nodes are increased by using Diameter Perimeter Model. Enhanced AODV-Local Repair Trial (EAODVLRT) protocol is implemented on NS2 network simulator. Simulations are performed to analyze and compare the behavior of proposed protocol (EAODVLRT) for varying parameters such as size of network, node load etc. Proposed protocol has been compared with the existing AODV-LRT in terms of routing load, Data delivery ratio.
\end{abstract}

\section{KEYWORDS}

AODVLRT, MANET, Local Repair ADHOC network \& Perimeter routing.

\section{INTRODUCTION}

A wireless network is a rising technology that will allow users to access services and information electronically, irrespective of their geographic position. Wireless communication network is a collection of independent devices connected to each other. Some of the advantages of wireless network are it is easily deployable and flexible in nature as compared to wired networks. There are two approaches for wireless communication that is centralized cellular network and decentralized approach (adhoc network). In centralized cellular network each mobile unit is connected to one or more fixed base stations, so that a communication between two mobile stations requires involving one or more base stations. In decentralized approach there may be situations where radio network units or nodes, move in terrain where line-of-sight communication rarely is possible between all nodes and where pre-deployed infrastructure cannot be guaranteed. A mobile ad hoc network (MANET) (Fig. 1) each device (nodes) is dynamically self organized in network without using any pre-existing infrastructure.

DOI : $10.5121 /$ ijwmn.2012.4216 
International Journal of Wireless \& Mobile Networks (IJWMN) Vol. 4, No. 2, April 2012

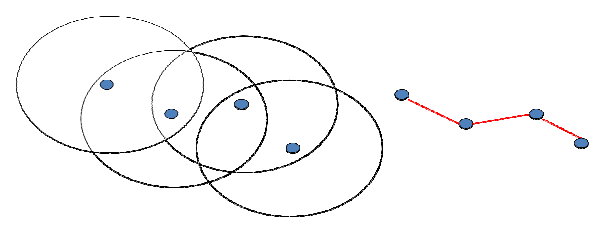

Fig.1 Mobile Ad-Hoc Network

The primary challenge in building a MANET is equipping each device to continuously maintain the information required to properly route traffic. Such networks may be operated by themselves or may be connected to the larger Internet. MANETs are a kind of wireless ad hoc networks that usually have a routable networking environment on top of a link layer ad hoc network. The mobility of nodes in Mobile Ad Hoc networks (MANETs) causes frequent changes of network topologies making routing in MANETs a challenging task.

Motivation for current work is that Ad hoc network allows all wireless devices within range of each other without involving any central access point and administration. Increase in number of nodes degrades the performance of large ad hoc network that makes the design of routing protocols more challenging. There are many simulation study has been done so far for the routing protocols. This paper has been organized as follows: In the following section we briefly review the two protocols AODVLRT (AODV with Local Repair Trials) [1] and AODV [2].

The performance metrics are described based on the comparison of the protocols. Next to this a simulation model has been explained on which basis results are obtained and graphs are generated to compare and analyze the results with the help of performance metrics. We have presented the simulation based comparative performance analysis of routing protocol and finally concluded that the enhanced version of AODVLRT protocol is better under certain traffic conditions and scenarios. The main motivation behind the current work is enhancing the AODVLR protocol by reducing the routing overhead. There will be impact on performance for low bandwidth in wireless link if high routing load is there.

The main objective of this paper is to enhance AODVLRT (AODV Local Repair Trial) protocol by minimizing flooding using perimeter routing. In the previous implementation [1] throughput increases with the increase of routing overhead but, in this paper a novel method is proposed to reduce the two parameters i.e. controlling overhead and increasing throughput are the major areas of focus. The remainder of this paper is organized as follows. In section 2, a short introduction to AODV, AODVLR (AODV Local Repair), AODVLRT (AODV Local Repair Trial) is presented. In Section 3, we suggest an improvement of AODVLRT by implementing perimeter routing. Section 4 describes the simulation model adopted, and then a detailed simulation is performed to evaluate the performance of the Enhanced AODVLRT (EAODVLRT). Conclusions are presented in section 5.

\section{AODV AND ROUTE REPAIR}

\subsection{Overview}

Ad hoc on demand distance vector routing is an on demand approach of route finding. Routing can be done when source nodes sends the packet for transmission. AODV differs from the other on-demand routing protocols is in a way that it uses a destination sequence number to determine an up-to-date path to the destination but it doesn't broadcast update information in the network. But in this case the entire topology had being change in the network periodically [3]. Like the on-demand routing AODV source node also floods the route request packet in the network. The routing can be done at the intermediate node is established by comparing the sequence number 
International Journal of Wireless \& Mobile Networks (IJWMN) Vol. 4, No. 2, April 2012

of source and destination in the route request packets. If a route request is received multiple times, duplicate copies must be discarded. AODV protocol generally uses mobile sensor nodes in a multi- hop wireless network which is quickly adaptable for dynamic link condition, it uses unicast route (asymmetric in nature) to the destination. In AODV the destination node chooses one among all possible discovered routes [3].

Major advantage of AODV is that the connection setup delay is much less than other protocol [3]. While the drawback is that the inconsistent routes are also discovered. The periodic beaconing also leads to unnecessary bandwidth consumption.

\subsection{Local Repair AODV}

AODV is a popular on demand routing protocols for mobile adhoc network. The major drawback which the AODV suffer lots of link failure [4] with the failure of single node the whole route is rejected AODV can basically work in two repair techniques:
(1) Source Repair
(2) Local Repair

The wireless multi-hop networks are suffered from link failures so, it is necessary to repair the routes. In the Ad-hoc On Demand Distance Vector (AODV) protocol, routes can either be repaired by re-establishing a new route from starting to the source node (Source Repair), or they can be locally repaired by the node that detects the link break along the end-to-end path (Local Repair) [5].AODV is one of the major reactive protocols which mean route discovery are initiated on demand. Once a route is discovered between two nodes, data transfer occurs until the route is broke due to node movement or inference due the erroneous nature of wireless medium. When a route failure happens between two nodes route maintenance can initiated. The upstream node of failure tries to repair that route and this process called local repair [6]. In the AODV routing protocol is reactive protocol which means route discovery can be done on demand, if the particular node failure can occur then the whole routing can be done. To avoid this problem local repair technique is being added by AODV and new protocol had being generated called as AODVLRT [1].

\subsection{AODV Local Repair Trial (AODVLRT)}

AODVLRT is modification of local repair algorithm used in the route maintenance of the AODV routing protocol. The AODVLRT mainly reduces the routing message overhead resulted from the original AODV local repair algorithm [1]. The enhancement leads to higher throughput and lower latency when compared to AODV. Major difference between AODVLR and AODVLRT is just one trial to find a repair to the route by broadcasting RREQ packet with TTL come from below equation which is taken from [1].

$$
T T L=\operatorname{Max}\left(0.5 \times N_{H}, T T L_{M N R}\right)+T T L_{L A}
$$

Where,

TTL $L_{M N R}$ : the last known hop count from the upstream node of the failure to the destination. $\mathrm{TTL}_{\mathrm{LA}}$ : constant value

$\mathrm{N}_{\mathrm{H}}$ : the number of hops from the upstream node of the failure to the source of the currently undeliverable packet. 
International Journal of Wireless \& Mobile Networks (IJWMN) Vol. 4, No. 2, April 2012

\section{IMPROVEMENT TO THE STANDARD AODVLRT}

Routing can be done from source node to the destination node by flooding the route request packet. It employs destination sequence numbers to identify the most recent path. The destination sequence number is created by the destination that is included along with any route information it sends to the requesting nodes. Destination sequence number gives the choice between two routes; a requesting node is required to select the one with the greatest sequence number. During the process of routing failure, of a node causes the whole route to be rejected. To overcome this, repairing technique can be used. The behavior of AODV in case of link failure as defined in [1, 4]. In EAODVLRT flooding can be minimized by combining the concept of perimeter routing [7].

\subsection{System Model}

We represent a wireless ad hoc network by a graph $G=(V, E)$ where $V$ is the set of vertices which represents mobile nodes and $E$ subset of $V^{2}$ the set of edges between these vertices. An edge exists between two nodes if they are able to communicate to each other, that is two nodes $\mathrm{u}$ and $\mathrm{v}$ can communicate if they are in communicating radius of each other. If all nodes have the same range $\mathrm{R}$, the set $\mathrm{E}$ is defined as:

$$
E=\left\{(u, v) \in V^{2} \mid u \neq v \& d(u, v) \leq R\right.
$$

$\mathrm{D}(\mathrm{u}, \mathrm{v})$ being the Euclidean distance between $\mathrm{u}$ and v. we also define neighborhood set $\mathrm{N}(\mathrm{u})$ of the vertex $u$ as

$$
N(u)=\{v \mid(\mathrm{u}, \mathrm{v}) \in E\}
$$

This system environment also makes following assumptions:

- Nodes are being connected with the symmetric link properties.

- The set of all nodes in the system is denoted as $\mathrm{M}=\{\mathrm{M} 1, \mathrm{M} 2 \ldots \mathrm{MN}\}$, where $\mathrm{N}$ is the total number of nodes in the network.

- Nodes are dynamic in nature, so that TTL_Threshold value should be initialized dynamically.

- Intermediated nodes between sources to destination nodes can be 40 for using network diameter model.

- Time to live increment (TTL_increment) which is assumed two and TTL_LA values are pre initialized are static. Every node has different battery power.

- Mobility random mobile model is used with predefined pause time.

\subsection{EAODVLRT Algorithm}

- In the algorithm, an AODV-node informs its neighbours about its own existence by constantly sending "hello messages" at a defined interval.

- Discovery of neighboring nodes is done by perimeter routing protocol. Through perimeter routing, the sender can only broadcast the RREQ packets to the outer boundary in counter clockwise direction. A RREQ contains the sender's address, the address of the source node and the maximum sequence number received from the node which exists.

- If the source cannot find the destination then that route can be discarded, again new route can be searched by using the local repair techniques. 
International Journal of Wireless \& Mobile Networks (IJWMN) Vol. 4, No. 2, April 2012

- Local Repair will increase the routing protocol performance. The major idea is controlling messages from neighbour nodes; this can be done by minimizing flooding.

- In the AODV model, the inquiry about the particular route from source to destination by default is 2 but it can be increased to 7 times in EAODVLRT.

- The receiving node checks whether it has a route to the particular node. If a route exists and the sequence-number for new received route is higher than the existing route then it accepts the new route. The node replies to the requesting node by sending a route reply (RREP). On the other hand, if a route does not exist then the receiving node sends a RREQ by itself in order to find a route for the requesting node.

- If the original node does not receive an answer within a time-limit the node can assume that the source nodes are unreachable. Then the request was sent to all neighbouring nodes which are easily separated by the sequence numbers. Nodes along the route will keep their routing table updated. Otherwise, the nodes will discard the entries after a particular time. To be sure that the route still exists, the sender has to keep the route alive by periodically sending packets. The broken link will send the error message (RERR) to the closest node, so that the nodes which identified the broken link can start to search for a new route.

\subsection{Description EAODVLRT Algorithm}

In a wireless network, a route is searched from source to destination by broadcasting the route request message by the source node. The broadcasting can be done by using the perimeter routing $[7,8]$. Through the perimeter routing the sender can only broadcast the RREQ packet to the outer boundary in counterclockwise direction. When broadcasting is done in perimeter mode, overlapping of links between the neighbor nodes can be avoided by constructing a planer graph using RNG or GG [9, 10]. By using this procedure flooding can be minimized. After flooding, destination node replies with the corresponding route reply message. During this routing process intermediate nodes create routing table entries for both source and destination nodes. There by creating bidirectional end-to-end route. The major drawback of AODV is that it suffers with a lot of link failures [11]. To overcome this problem local repair technique can be used.

In local repair technique once a route is discovered between two nodes, data transfer occurs until the route is broken due to node movement or inference caused due to the erroneous nature of wireless medium. Route maintenance is initiated when a route failure happens between two nodes. The upstream node of failure tries to repair that route and this process is called local repair.

The proposed protocol uses the local repair technique. AODV can broadcast the RREQ message in the network. The RREQ message is re-broadcasted by the other nodes in the network until it can reach the destination. The route is searched for two times by default in the previous AODV model but in EAODVLRT protocol it can be maximized to seven times in the particular route. In mobile ad hoc network, the mobility of each nodes can be assumed as random way point mobility model [14] with the static pause time. Due to the dynamic nature of the network, the new node may enter to the network that will set the route to the destination i.e. the number of searches to destination for particular route can be increased by seven times. 
International Journal of Wireless \& Mobile Networks (IJWMN) Vol. 4, No. 2, April 2012

In the mobile ad-hoc network there is wireless connection between mobile nodes with limited bandwidth [13]. So if the flooding increases in the network the bandwidth will be spread into the network. Then the bandwidth utilization of the nodes in the wireless network will be increased. Here, our aim is to minimize the Flooding; automatically decreasing bandwidth utilization.

In the EAODVLRT algorithm Flooding can be minimized by the local repair technique.

Local repair is a technique used to repair a broken route locally on the upstream nodes of the link failure to the destination no farther than TTLMNR. To repair the link failure, the upstream node broadcasts RREQ packet after increasing the destination sequence number [1]. The TTL value used in RREQ packet is set by the following values:

$\mathrm{TTL}=\operatorname{Max}(0.5 * \mathrm{NH}, \mathrm{TTLMNR})+\mathrm{TTLLA}$

Where:

TTL $=$ Time to live is the constant value which will limits the life span of network, which is generally being used for increasing the number of intermediate nodes in the thesis we had set as 40.

TTLMNR = the last known hop count from the upstream node of the failure to the destination.

TTLLA $=$ constant values which is assumed to be five.

$\mathrm{NH}=$ the number of hops from the upstream nodes of the failure to the source of the currently undelivered packet.

TTL_increment $=$ when a route failure happens, the upstream node increments the destination sequence number by two.

After the upstream node broadcasts the RREQ packet, it waits for the discovery period to receive RREP packets in response to the RREQ packet. When the destination or an intermediate node that has a fresh route to the destination receives the RREQ packet, a RREP packet will be forwarded towards the upstream node. If discovery period finishes and the upstream node didn't receive a RREP for that destination, it transmits a RERR message for that destination to the source. On the other hand, if the upstream node receives one or more RREP packets during the discovery period, it first compares the hop count of the new route with the value in the hop count field of the invalid route table entry for that destination. In the case of the hop count of the newly determined route to the destination is greater than the hop count of the previously known route, the upstream node transmits a RERR message for that destination towards the source, with 'N' bit set. Finally, the upstream node updates its route table entry for that destination.

In Fig. 2 shows the generalized work flow diagram of EAODVLRT which will show the above steps in the diagrammatical form. 
International Journal of Wireless \& Mobile Networks (IJWMN) Vol. 4, No. 2, April 2012

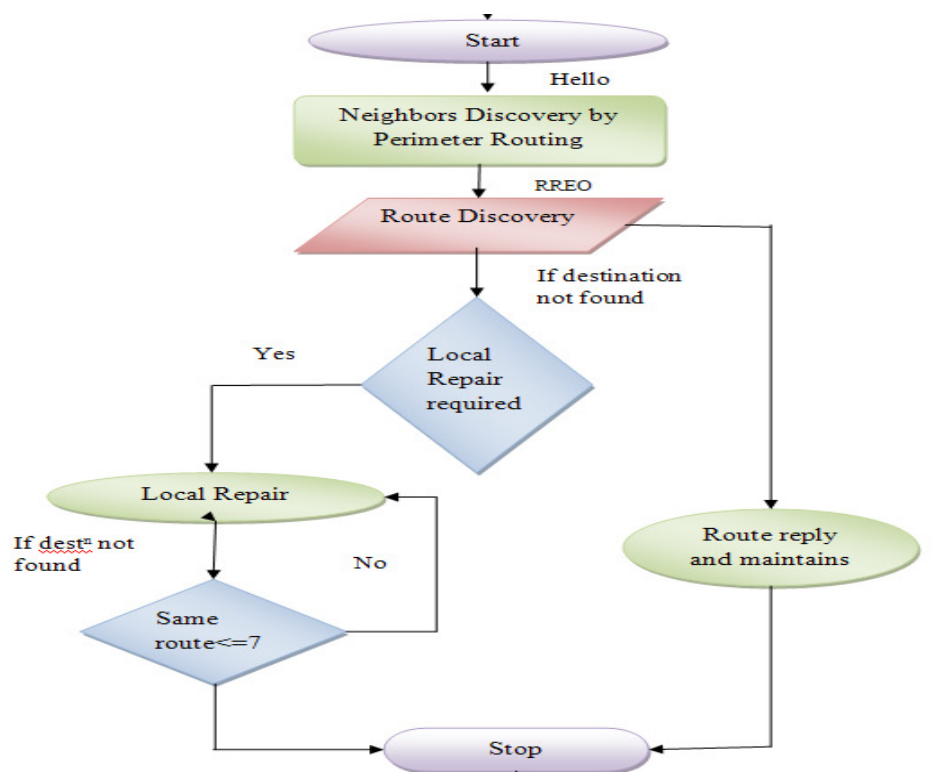

Fig.2 Generalized work flow diagram of EAODVLRT

In AODV TTL_Threshold value should be static in nature. But, in ad hoc network each and every node is dynamic in nature, so every time the topology changes. Therefore, TTL_Threshold value can change every time. By applying this concept TTL_Threshold value can be dynamic in nature.

Once a network is in perimeter method, it prevents the links between nodes from being overlapped by constructing a planar graph using RNG or GG [21, 22]. In this procedure, a packet is forwarded from a start node $\mathrm{F}$ to its destination node $\mathrm{D}$ guided by the planar graph in clockwise direction as shown in Fig. 3

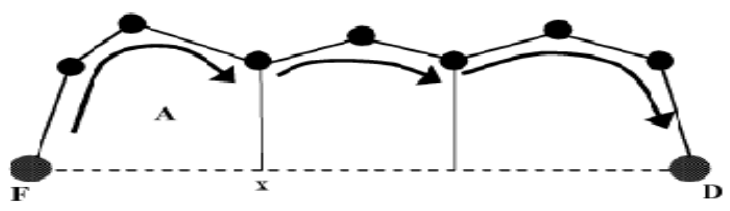

Fig.3 Perimeter forwarding method

In the network perimeter model a packet traverses successively through closer faces of a planar sub graph of the full radio network connectivity graph, until reaching a node closer to the destination.

\subsection{Perimeter Algorithm}

The main idea behind perimeter forwarding is to forward the packets using the right hand rule across the faces in the planar graph that intercept the line $L_{p} D$ (Fig.4). The algorithm used for perimeter forwarding [15] is iven below: 


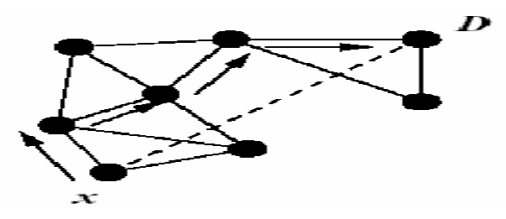

Fig.4 Perimeter Forwarding [8]

The PERIMETER-INIT-FORWARD [23] algorithm forwards a packet $\mathrm{p}$ to a node $\mathrm{a}_{\min }$, where, $\left(a_{\min }\right.$, self) is the first edge encountered countered clockwise from the line $L_{p} D$ by perimeter routing where $\mathrm{p}$ is packet which are send to the destination .

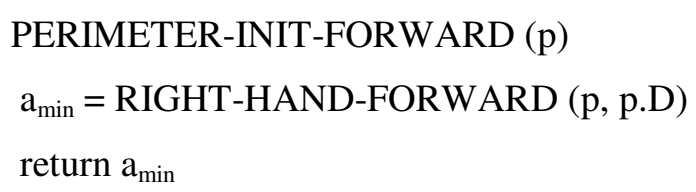

The RIGHT-HAND-FORWARD [16] algorithm implements the right-hand rule method for traversing polygons, which in our case are the faces in the planar graph. The time complexity of the algorithm is $\mathrm{O}(\mathrm{cN})=\mathrm{O}(\mathrm{N})$, where $\mathrm{N}$ is the number of neighbors in the planar graph and $\mathrm{c}$ is the time it takes to do a NORM2 operation. NORM can be considered as a constant operation,

Since the range of the arc of the tangent is $(-\pi / 2, \pi / 2)$.

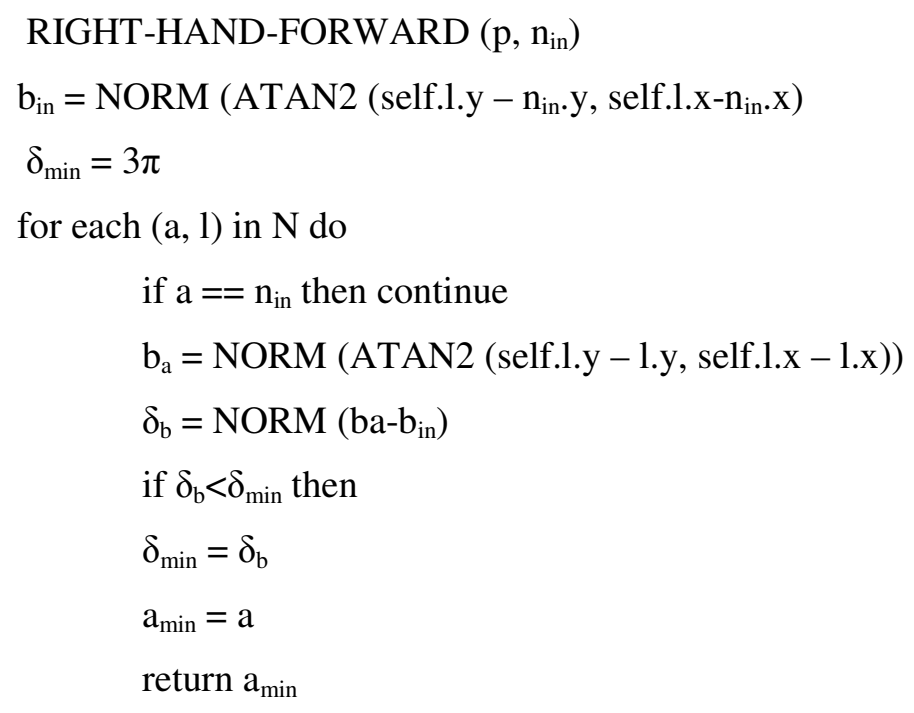

If the next edge (self.a, $t$ ) in the network is found counter clockwise direction by RIGHTHAND-FORWARD intercepts $\mathrm{L}_{\mathrm{p}} \mathrm{D}$, EAODVLRT updates the packet $\mathrm{e}_{0}$ field and instead of selecting node $t$ to forward the packet to, it selects the next edge counter clockwise from (self.a, t). We will compare the performance of AODVLRT with the proposed protocol (EAODVLRT) under such conditions for parameters namely throughput, average end to end delay, packet loss, packet delivery fraction. 
International Journal of Wireless \& Mobile Networks (IJWMN) Vol. 4, No. 2, April 2012

\section{SIMULATION MODEL AND RESULTS}

In this section, a series of simulation experiments in NS2 [17] network simulator will be conducted to perform an evaluation analysis on the performance ability of EAODVLRT with the discussed mechanism. We choose ad hoc on demand distance vector (AODV) routing algorithm as the underlying protocol for our base case simulation. AODV is a source initiated reactive (on-demand) protocol, which initiates a route discovery wherever a node requires a path to a destination. Following matrix is used to analyses the performance of EAODVLRT algorithm.

\subsection{Simulated Network Scenario and Model}

We consider a network of nodes placing in various arrangements within a $1500 \times 600 \mathrm{~m}$ area. The performance of AODV and EAODVLRT is evaluated by keeping the network size (number of mobile nodes) constant and varying the maximum speed of the nodes. The values of AODVLRT have been taken from base paper [1] which we have implemented. Table 1 show the simulation parameter used in the evaluation.

TABLE 1 Simulation Environment

\begin{tabular}{|l|l|}
\hline Dimension of simulated area & $1500 \times 600 \mathrm{~m}$ \\
\hline Simulation Time & $300 \mathrm{sec}$ \\
\hline Mobile Nodes & 50 \\
\hline Transferring Mode & Unicast \\
\hline Pause time & $\begin{array}{l}0,50,100,150,200,250,300 \\
(\mathrm{~m} / \mathrm{s})\end{array}$ \\
\hline Traffic & CBR \\
\hline Packet Size & 1024 bytes \\
\hline Routing Protocols & AODV, Enhanced AODVLR \\
\hline Transport Layer agent type & TCP \\
\hline Maximum speed & 35 \\
\hline Transmission range & $250 \mathrm{~ms}$ \\
\hline Mobility & Random \\
\hline Bandwidth & 1 megabits/sec \\
\hline
\end{tabular}

\subsection{Performance Metrics}

Analysis of routing protocols for parameters like packet delivery fraction, average end to end delay, packet loss, routing overhead will be done. The parameters are defined in the following section.

- Packet Delivery Ratio: It is the ratio of data packets delivered to the destinations to those generated by the CBR sources is known as packet delivery ratio. 
International Journal of Wireless \& Mobile Networks (IJWMN) Vol. 4, No. 2, April 2012

Packet Delivery Ratio $=$ packet received $/$ delivered packets

- Average End To End Delay: Average end-to-end delay is delay of data packets. Buffering during route discovery latency, queuing at interface queue, retransmission delays at the MAC and transfer times may cause this delay. Once the time difference between every CBR packets sent and received was recorded, dividing the total time difference over the total number of CBR packets received gave the average end-to-end delay for the received packets. Lower the end to end delay better is the performance of the protocol.

Average End to End Delay= Total end to end delay/number of packets received

- $\quad$ Routing Overhead: Routing overhead is the number of routing packets transmitted per data packet delivered at the destination. Each hop-wise transmission of a routing packet is counted as one transmission. The first two metrics are the most important for best-effort traffic. The routing load metric evaluates the efficiency of the routing protocol.

Routing Overhead $=$ Total routing packets $/$ transmitted data packets .

- Average Throughput: The average number of packets received per amount of time (from the first packet sent to the last packet received).

Average Throughput $=$ Total received packets $/$ simulation time

- Packet Loss: It is defined as the difference between the number of packets sent by the source and received by the sink. The routing protocol forwards the packet to destination if a valid route is known; otherwise it is buffered until a route is available. There are two cases when a packet is dropped: the buffer is full when the packet needs to be buffered and the time exceeds the limit when packet has been buffered. Lower is the packet loss better is the performance of the protocol.

Packet Loss $=$ sent packets- received packets.

Note, however, that these metrics are not completely independent. For example, lower packet delivery fraction means that the delay metric is evaluated with fewer samples. In the conventional wisdom, the longer the path lengths, the higher the probability of a packet drops. Thus, with a lower delivery fraction, samples are usually biased in favor of smaller path lengths and thus have less delay.

\subsection{Simulation Results and Technical Analysis}

EAODVLRT simulation is based on the same environment as for AODV simulation in NS2. Because mobility is the key reason for packet losses, we design the scenarios for comparing the performance of AODV and EAODVLRT based on random mobility model. As indicated in Section 4.A, the simulation environment can be determined by maximum movement speed and the pause time during simulation. This section presents the simulation results and their analysis for 50 nodes network simulation scenario on a rectangular area $1500 * 600 \mathrm{~m}^{2}$.

\section{Routing message overhead}

The routing message overhead resulted from AODV, AODVLRT [5] and EAODVLRT routing protocols has been presented in Fig. 5. From Fig (5), it could be noticed that EAODVLRT has 
International Journal of Wireless \& Mobile Networks (IJWMN) Vol. 4, No. 2, April 2012

lower routing message overhead by on average $42 \%$ less that the AODV which had been calculated from TABLE 2. For the comparisons purpose values of AODVLRT has being taken from [5]. This result demonstrates the effect of local repair trial by using perimeter routing in EAODVLRT on reducing routing message overhead. This is due to the fact that when the node mobility is increased, the frequency of topology changes is also increased. This can potentially trigger more new route maintenance processes, resulting from the broken routes. As a consequence, larger numbers of RREQ packets are generated and disseminated.

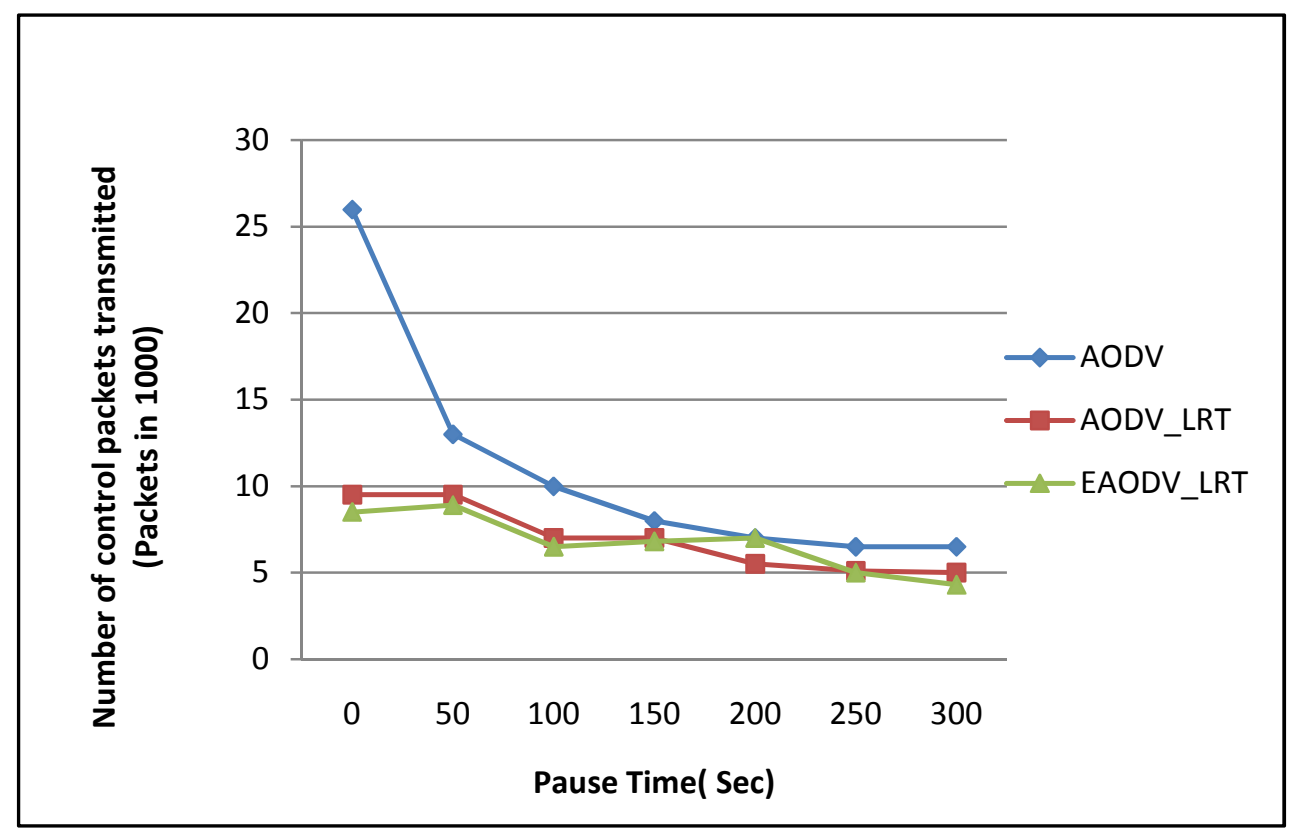

Fig.5 Routing Overhead Analysis

This result demonstrates the effect of local repair trial using perimeter routing in EAODVLRT on reducing routing message overhead. This is due to the fact that when the node mobility is increased, the frequency of topology changes is also increased. As a consequence, larger numbers of RREQ packets are generated and disseminated. Result shows our EAODVLRT routing overhead is minimize as compare to AODV routing protocol. This performance behavior is expected since increasing the offered load leads to an increase in the number of source nodes that initiate route discovery operations.

\section{Throughput}

The throughput resulted from AODV, AODVLRT and EAODVLRT has been presented in Fig 6. It can be found that EAODVLRT has higher throughput than AODV routing protocol by an average $1.66 \%$ which is a small increase which had being calculated from TABLE 3 . It can be found that EAODVLRT has higher throughput than AODV routing protocol by an average $1.66 \%$ which is a small increase. This result demonstrates that the effect of the modifications in EAODVLRT doesn't appear in small sized networks. The number of packets dropped or left wait for a route affect the throughput as the increase in the number of packets dropped or left wait for a route reduce the throughput. The number of packets dropped or left wait for a route reduce the throughput. The numbers of packets dropped or left wait for a route affected by the success of local repair in repairing a failed route, where the number of packets dropped or left wait reduced as the percentage of success local repair attempts increased. 


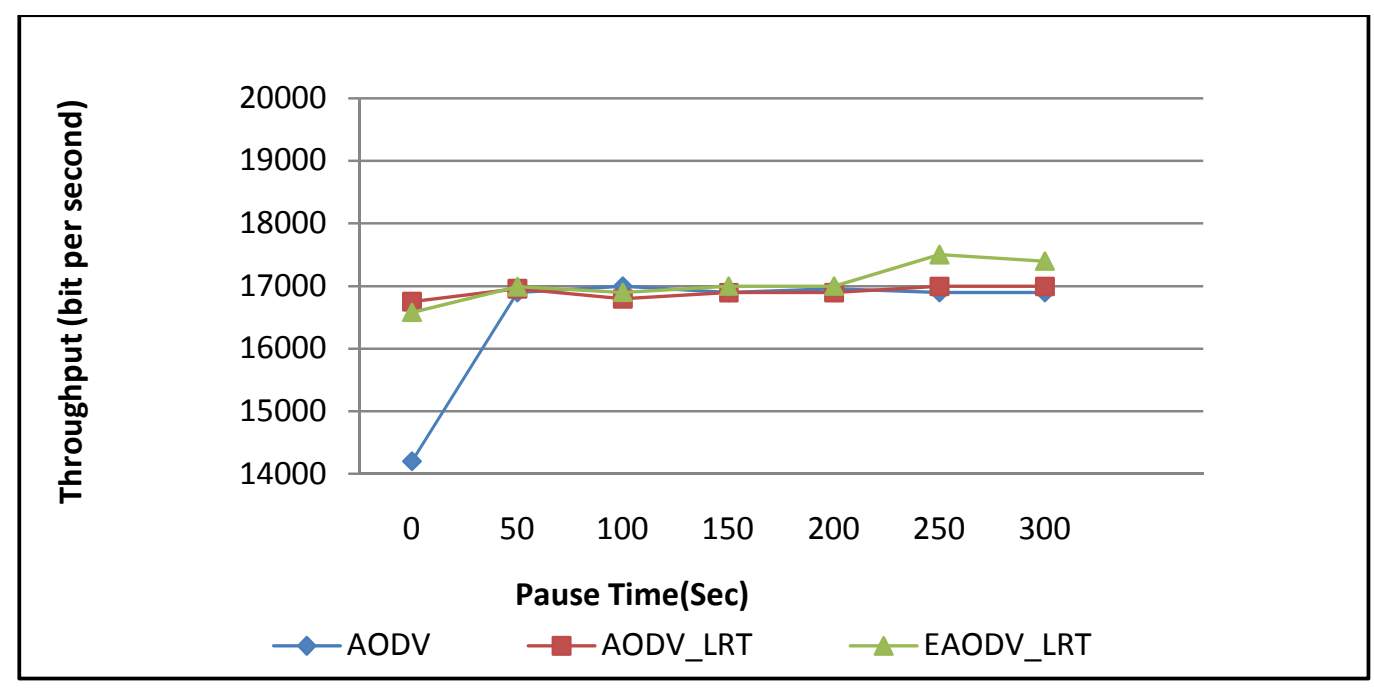

Fig.6 Throughput Analysis

This result returns to that local repair in EAODVLRT acts in trials by broadcasting first RREQ packet with TTL $=$ LR_TTL_START (equal to 2 in experiment). This reduces the routing overhead which by its turn resulted in increasing throughput. On the other side, local repair in AODV broadcasts RREQ packet once with TTL as in AODV local repair in AODV which resulted in higher routing message overhead which led by its turn to reduce the throughput.

\section{Average End to End Delay}

Fig 7 presents a graph of packet delivery ratio of AODV and EAODVLRT routing protocols. It is clear that EAODVLRT gives average packet delivery ratio is higher than the AODV by $71.98 \%$ which had been calculated from TABLE 4. It is clear that EAODVLRT gives average end to end delay higher than the AODV by on average $28 \%$ with difference. The result demonstrates the high effect of local repair trial in EAODVLRT on the delay of the small size of networks which resulted from broadcasting RREQ with TTL as in local repairs. The figure shows that when nodes pause time increases, the end-to-end delay of data packets also increases. This is because the paths between sources and required destinations frequently changed and established. However, among all maximum pause time the AODV performs better, followed by EAODVLRT and AODVLRT.

The increase in the number of broken links will led to increase the delay of transferring packets on a route until finding a repair to the route. The number of broken links affected by the route length as longer routes means the higher chances for broken links. In the same time, the number of broken links affected. 
International Journal of Wireless \& Mobile Networks (IJWMN) Vol. 4, No. 2, April 2012

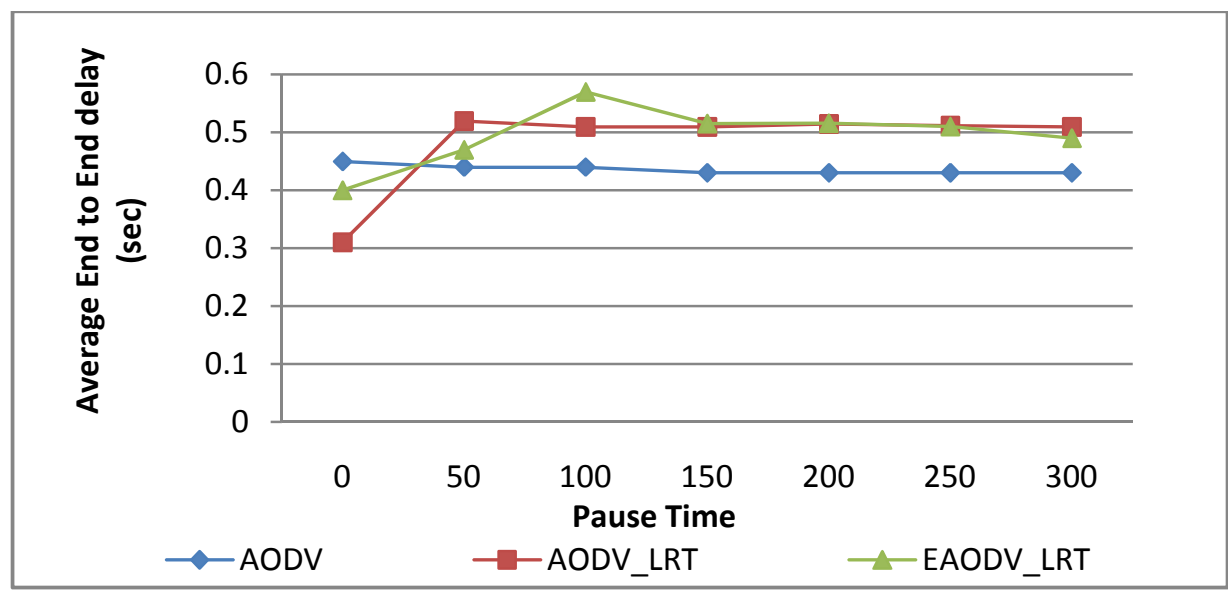

Fig. 7 Average End to End Delay

\section{Packet Delivery Ratio}

Fig 8 presents a graph of average end to end delay of AODV, AODVLRT and EAODVLRT routing protocols. It is clear that EAODVLRT gives average end to end delay higher than the AODV by on average $28 \%$ with difference, which had being calculated from TABLE 5 . It is clear that EAODVLRT gives average packet delivery ratio $71.98 \%$. If we look at this graph, which is for packet delivery ratio of both the protocols, the packet delivery by EAODVLRT is better than AODV. Results shown above concluded that EAODVLRT has packet delivery ratio which is better as compared to AODV Protocol.

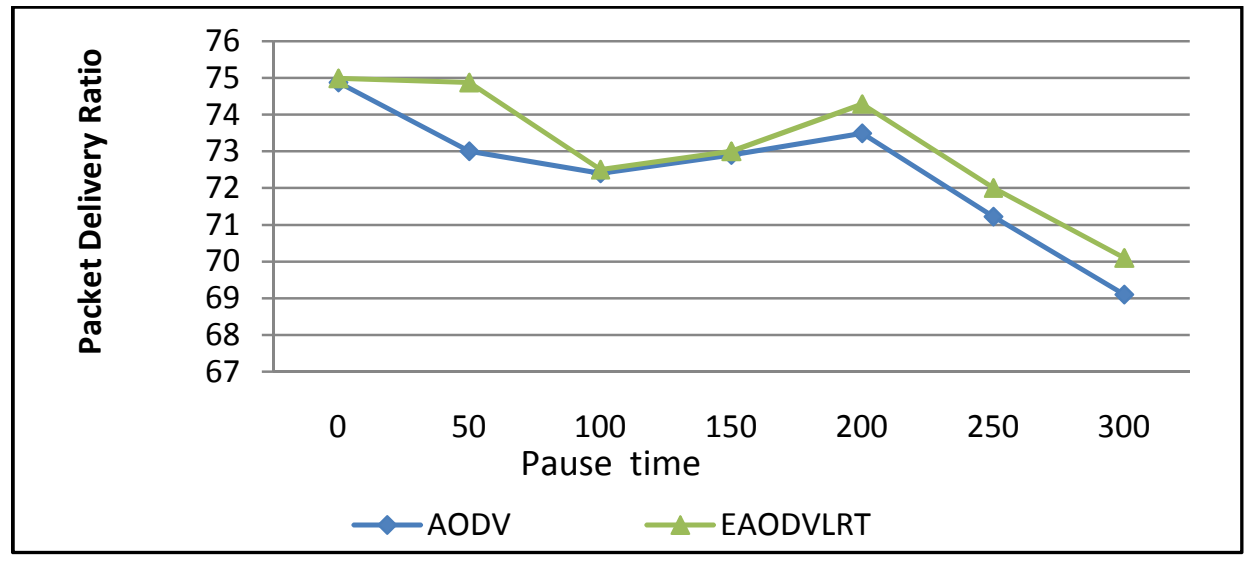

Fig 8 Packet Delivery Ratio

\subsection{Overall Summary of AODV and EAODVLRT}

The new protocol EAODVLRT perform better than that of AODV routing protocol in terms of "Throughput", "Packet Delivery Ratio", "Routing Overhead" and "End to End Delay". In case of traditional AODV the graph depicts a similarity with EAODVLRT but the values of overall throughput remain less as compared to the EAODVLRT by $1.66 \%$.

Here TABLE 2 shows the comparisons of between AODV and EAODVLRT. 
International Journal of Wireless \& Mobile Networks (IJWMN) Vol. 4, No. 2, April 2012

TABLE 2 Comparisons for Throughput

\begin{tabular}{|c|c|c|}
\hline Pause Time & AODV & EAODVLRT \\
\hline 0 & 14200 & 16580 \\
\hline 50 & 16900 & 16900 \\
\hline 100 & 17000 & 17000 \\
\hline 150 & 16900 & 17000 \\
\hline 200 & 16955 & 17500 \\
\hline 250 & 16900 & 17400 \\
\hline 300 & 16900 & \\
\hline
\end{tabular}

TABLE 3 Comparisons for Routing Overhead

(Packets is 1000)

\begin{tabular}{|c|c|c|}
\hline Pause Time & AODV & EAODVLRT \\
\hline 0 & 26 & 8.5 \\
\hline 50 & 13 & 6.5 \\
\hline 100 & 10 & 6.8 \\
\hline 150 & 8 & 7 \\
\hline 200 & 7 & 5.3 \\
\hline 250 & 6.5 & 6.5 \\
\hline 300 & & \\
\hline
\end{tabular}

Based on Fig. 7 with the variation in pause time, EAODVLRT is performed better in delivering data packet to the destination than AODV. However, when pause time increased, the packet delivery ratio shows the variation on both AODV and EAODVLRT due to the random way point mobility model. Here, TABLE 4 shows the results comparison between both the protocols that is AODV and EAODVLRT.

TABLE 4 Packet Delivery Ratios

\begin{tabular}{|c|c|c|}
\hline Pause Time & AODV & EAODVLRT \\
\hline 0 & .45 & .47 \\
\hline 50 & .44 & .57 \\
\hline 100 & .44 & .515 \\
\hline 150 & .43 & .516 \\
\hline 200 & .43 & .51 \\
\hline 250 & .43 & .49 \\
\hline 300 & .43 & \\
\hline
\end{tabular}


International Journal of Wireless \& Mobile Networks (IJWMN) Vol. 4, No. 2, April 2012

The Fig. 8 shows that the performance of AODV routing protocol in terms of End to End Delay increases by increasing pause time with random mobility model. EAODVLRT takes less time to deliver the packets. Therefore the optimal delay is achieved but in case of traditional AODV the value of End to End Delay remains less. TABLE 5 shows the average end to end delay of two protocols.

TABLE 5 End to End Delay

\begin{tabular}{|c|c|c|}
\hline Pause Time & AODV & EAODVLRT \\
\hline 0 & 74.88 & 74.99 \\
\hline 50 & 73 & 74.87 \\
\hline 100 & 72.4 & 72.5 \\
\hline 150 & 72.9 & 73 \\
\hline 200 & 73.49 & 74.29 \\
\hline 250 & 71.22 & 72 \\
\hline 300 & 69.1 & 70.1 \\
\hline
\end{tabular}

\section{CONCLUSIONS}

This paper presents a novel approach to minimize routing overheads of AODVLRT. It also analysis enhanced AODVLRT with the existing local repair technique. This approach based on perimeter routing is used to minimize flooding process in EAODVLRT. In this paper we considered the mobile adhoc network routing protocol. Then this work analyzed the issues regarding AODV Local Repair in MANETs while exploring some existing Repair (AODVLRT) technique in literature. This technique consists of three modules. First, broadcasting and can be done by using the perimeter routing. Secondly, flooding is minimized by using local repair method and lastly, number of intermediate nodes from particular source to destination has being increased. This thesis is improved the performance of existing on-demand routing (AODV) protocols by reducing the RREQ overhead during the rout discovery operation. For its implementation and the analysis outcomes NS2 network simulator is used. For analyzing the performance of proposed schema (EAODVLRT) with the existing AODV comparisons had being done. The simulation results show that proposed schema gives the best performance in terms of packet delivery ratio, throughput and number of overhead which is used to compare the performance of these techniques.

\section{REFERENCES:}

[1] Maged Salah Eldin Solimana, Sherine Mohamed Abd El-kaderb, Hussein Sherif Eissac, Hoda Anis Barakad "New adaptive routing protocol for MANET" Ubiquitous Computing and Communication Journal, 2006.

[2] C.E Perkins, E. Belding-Royer and S. Das, "Ad Hoc on-Demand Distance Vector (AODV) Routing" RFC 3561, 2003. 
International Journal of Wireless \& Mobile Networks (IJWMN) Vol. 4, No. 2, April 2012

[3] Tao Yang, Leonard Barolli, Makoto Ikeda, Fatos Xhafa and ArjanDurresi, "Performance Analysis of OLSR Protocol for Wireless Sensor Networks and Comparison Evaluation with AODV Protocol" IEEE, 2009.

[4] Azzuhri S.R., Portmann M. and Wee Lum Tan "Evaluation of parameterized route repair in AODV" Signal Processing and Communication Systems (ICSPCS), 2010, 4th International Conference on1315 Dec. 2010.

[5] Mznah A. Al-Rodhaan \& Abdullah Al-Dhelaan, "Efficient Route Discovery Algorithm for MANETs" Fifth IEEE International Conference on Networking, Architecture and Storage, 2010.

[6] Joo-Sang Youn, Ji-Hoon Lee, Doo-Hyun Sung, "Quick Local Repair Scheme using Adaptive Promiscuous Mode in Mobile Ad Hoc Networks" journal of networks, vol. 1, no. 1, may 2006.

[7] Kyung-Bae Chang, Dong-Wha Kim, and Gwi-Tae Park "Routing Algorithm Using GPSR and Fuzzy Membership for Wireless Sensor Networks" Springer-Verlag Berlin Heidelberg 2006, pp. 1314 1319, 2006.

[8] Brad Karp and H. T. Kung "GPSR: Greedy Perimeter Stateless Routing for Wireless Networks" Mobi Com 2000.

[9] G. Toussaint, "The Relative Neighborhood Graph of a Finite Planar Set Pattern Recognition" Vol. 12, No 4 (1980).

[10] K.Gabriel \& R. Sokal "A New Statistical Approach to Geographic Variation Analysis, Systematic Zoology”, Vol. 18 (1969), pp. 259-278.

[11] M. Jiang \& Y. C. Tay, "Cluster based routing protocol", IETF Internet Draft, draft-ietf-manet-cbrpspec-02.txt, 1999.

[12] Jagpreet Singh, Paramjeet Singh \& Shaveta Ran,"Enhanced Local Repair AODV (ELRAODV)" International Conference on Advances In Computing, Control, and Telecommunication Technologies, IEEE 2009.

[13] Mohd Izuan, Mohd Saad, Zuriati Ahmad Zukarnain, "Performance Analysis of Random-Based Mobility Models in MANET Routing Protocol", European Journal of Scientific Research ISSN 1450-216X Vol.32 No.4 (2009), pp.444-454.

[14] Ching-Wen Chen, Chuan-Chi Weng, "Bandwidth-based routing protocols in mobile ad hoc Networks" Springer Science+Business Media, LLC 2008, pp 240-268.

[15] Maria del Mar Alvarez-Rohena \& Chris Eberz, "Implementation and Analysis of GPSR: Greedy Perimeter Stateless Routing for Wireless Networks", IEEE, 2010.

[16] Karp, B., "Geographic Routing for Wireless Networks”, Ph.D. Dissertation, Harvard University, Cambridge, MA, October, 2000.

[17] NS2 manualhttp://www.isi.edu/nsnam/ns/doc/ns_doc.pdf.

[18] Liu Chao and Hu Aiqun, "Reducing the Message Overhead of AODV by Using Link Availability Prediction” Springer-Verlag Berlin Heidelberg, LNCS 4864, pp. 113-122, 2007.

[19] L. Nithyanandan, G. Sivarajesh and P. Dananjayan "Modified GPSR Protocol for Wireless Sensor Networks" IEEE, Vol. 2, No. 2, April, 2010.

[20] Tran, D.A. Harish Raghavendra "Congestion Adaptive Routing in Mobile Ad Hoc Networks" Parallel and Distributed Systems, IEEE Transactions onNov. 2006, pp $1294-1305$.

[21] Jagpreet Singh, Paramjeet Singh \&Shaveta Rani, "Enhanced Local Repair AODV (ELRAODV)" IEEE 2009. 